\title{
Realization of the Based on WAP Push SMS Gateway
}

\author{
Zhongling Gong ${ }^{1}$,Wenjie X $\mathbf{u}^{2}$ \\ 1, 2. Gas Group Co., LTD of ShenZhen City , 518049, china
}

\begin{abstract}
WAP PUSH can send message actively and in time, as well as thatit can Receive message at all time and place, therefore there are favourable application prospec1 The paper has simplely introduced thesystem frame, the push protocol and the push way of WAP PUSH,especially it has introduced the implementation method of the message gateway for WAP PUSH.
\end{abstract}

\author{
KEYWORDS \\ WAP PUSH \\ Protocol
}

Message gateway

\section{INTRODUCTION}

WAP PUSH (PUSH) technology is a kind of based on client/server mechanism, is sent to the client by the server will actively information technology. Compared with traditional (PULL) technology, the main difference is that a PUSH (PUSH) technology is take the initiative to send information to the client by the server, and PULL (PULL) technology is the client requests information actively. The advantage of PUSH was initiative and timeliness of information.

PUSH technology failed to great success in the Internet, there are a variety of reasons. Main reason is that the fixed net capital equipment, such as computers, to provide users with enough resources and capabilities to find information so users usually use it as a browse information window, rather than a passive information receivers. At the same time, fixed network users to the requirement of information accuracy than the timeliness requirements, thus PUSH technology failed to get a wide range of applications.

In mobile net, because of network bandwidth, high ability of mobile terminals and at his own expense standard, and many other restrictions, allowing users to find information by certain restrictions, if the important information in a timely manner actively pushed to the users of mobile devices will no doubt greatly convenient for the user. Mobile communication has the advantage of mobile devices can receive information anytime and anywhere can therefore PUSH technology in the mobile web, WAP PUSH PUSH technology and the combination of the mobile communication both foster strengths and circumvent weaknesses. WAP PUSH is the application of PUSH technology in the mobile network, it combines the general PUSH technology and the characteristics of the mobile network. Its system framework, fixed online use protocol and service mode, PUSH technology is different [1].

Copyright @ 2016 Zhongling Gong and Wenjie Xu

doi: $10.18686 /$ esta.v3i1.6

This is an open-access article distributed under the terms of the Creative Commons Attribution Unported License (http://creativecommons.org/licenses/by-nc/4.0/), which permits unrestricted use, distribution, and reproduction in any medium, provided the original work is properly cited.

\section{Introduction of WAP Push Technology}

\subsection{WAP PUSH system framework}

WAP PUSH framework including PUSH the Initiator (PI: PUSH Initiator) and PUSH the gateway (PPG: PUSH Roxy Gateway) and PUSH the customer (PC: PUSH Client) three functional parts. PI with the Internet and PC in the field of WAP, PI and WAP protocol used by the client is different, need to establish a protocol conversion gateway that PPG in the middle. PPG through $\mathrm{PUSH}$ access Protocol (PAP: PUSH access Protocol) communicate with PI, by pushing space transport Protocol (PUSH OTA: PUSH over - the - Air) for transferring data to the client PUSH information tasks.

PPG push architecture most of the work done, including a visit from the Internet to the mobile access, as well as the related certification, safety and the client control all work. PPG's main services include: 1) PI identification, authentication and access control; 2) To push content parsing, and on the basis of the data type definition (DTD) error detection and error correction; 3) Customer address and information transmission; 4) The PAP and PUSH between OTA protocol conversion; 5) To improve the efficiency of wireless channel of transmission, to compress information, compile processing, etc.

In addition, PPG can also through the alias mechanism to realize multicast and broadcast, the alias is mapped to a specific address multicast or broadcast operations, specific solution can be decided by system implementers. Different clients, its ability is different, PPG will be responsible for the response of PI customer ability to query request, so that the $\mathrm{PI}$ for different client structure suitable content format.

\subsection{Push agreement}

PAP is communication protocol $\mathrm{PI}$ between the PPG, it USES extensible markup language (XML) as a message description language, through a simple request and response mechanism to complete data transmission. PAP can in a variety of communication protocols (including hypertext transfer protocol (HTTP), simple mail transfer protocol (SMTP), etc.) on the implementation. And PUSH the OTA is running in wireless session protocol (WSP) on top of a simpler protocol layer, is responsible for data transmission from PPG to customer agent. 


\subsection{Push service mode}

WAP push agreement defines the Service instruction (SI: Service Indication) and Service loading (SL: Service Load) two services, to users and network operators more choice. There will be a new information service instruction is instructions and associated universal resource identifier (URI) push to the user, is selected by the user to process information immediately or after processing. Service load is the URI of a service delivery to the user, and then the client automatically using the PULL technology based on the URI to start the service. The difference between the two services is whether the user involvement in push information processing. SL to push the processing of information is transparent to users, and $\mathrm{SI}$ in indicating the user at the same time, the user, please choose for subsequent processing.

PUSH Can combined a site or a business link sent via SMS to support WAP PUSH on the phone, so users only need to read this message, open the links in the text, can direct access to the business. Therefore, WAP PUSH to realize the combination of the SMS and WAP, saved users find business time, convenient users directly to find and use the business that oneself like.

\section{Short message gateway}

Short message gateway (ISMG) is at the short message center (SMSC) and service provider (SP) between the equipment, it is the data exchange of two entities to provide safe, fast. Use SMPP protocol between the gateway and Short Message center (Short Message Peer to Peer, the Short Message point-to-point protocol), and using the CMPP protocol between SP (China Mobile Peer to Peer, China Mobile peer-to-peer protocol), so the Short Message gateway need completion protocol conversion, billing, routing, security, and network management, and other functions. Specifically, SMPP communication agent system mainly realize the gateway and the GSM short message center (SMSC) connection, ensure accurate to send and receive data, efficient and reliable data transmission. In order to meet the specification requirements of no more than $0.001 \%$ of the data packet loss rate, SMPP communication agent needs to support flow control. CMPP communications agency system mainly is the implementation and SP service provider connection, unlike SMPP communication agent system, because of the influence of the agreement, CMPP communications agency is the server side, waiting for SP connections, and SMPP communications agency is the client, need to take the initiative to connect the SMSC. Short message gateway system is the most complex processing in the gateway, it completes tasks include: to GNS (tandem gateway) query routing, routing table maintenance and protocol conversion and data distribution. Firewall system is mainly to provide security for the gateway system, it includes IP packet filtering, and authentication. SMS gateway billing system main form various billing billed, provide the basis for billing. Main business management system to complete the business statistics report, generate reports, to the user data for operators to add, modify, delete, and monitoring of the gateway system, query, operation and maintenance provide interfaces, and interface.

\section{Based on message gateway sent WAP PUSH}

There are two ways to send WAP PUSH , one is by PPG gateway, another is via SMPP protocol. The SMPP is a basic agreement, there are three main derived from this agreement in China : China mobile CMPP protocol, China unicom SGIP (ETIP on CDMA), as well as PHS SMGP. Sent by China mobile PPG gateway WAP PUSH has a long development cycle, the measurement process is relatively complex, etc, and using the CMPP protocol is based on SMS gateway for WAP PUSH send flexibility is higher, is relatively simple.

\subsection{WAP PUSH to send the implementation of the model}

This can be connected through the computer serial port on the GSM MODEM, use it to send mobile WAP PUSH. BLOCK pattern with very little now, TEXT mode only can sending ASCII, it can't send Chinese UNICODE code, whereas PDU mode to develop more complex, it needs to write a special function to convert TEXT to PDU format, but the PDU mode supported by all mobile phones, you can use any character set, it is also a mobile phone to the default encoding, so choose PDU mode send WAP PUSH.

\subsection{PDU Mode}

PDU mode Transmission WAP PUSH can use three encoding: 7-bit encoding, 8-bit encoding and UCS2 encoding. 7-bit encoding used to send ordinary ASCII characters, 8-bit codes are usually used to send data messages, UCS2 code for sending Unicode characters. Due to the realization of Chinese WAP PUSH transmission, so we chose to use UCS2 encoding, that Chinese Unicode code [2].

\subsubsection{UCS2 coding theory}

The so-called UCS2 coding, is a single character (1-2 bytes) according to the provisions of ISO / IEC10646, the transition to 16-bit Unicode wide characters. ' 0 ' upcoming single character conversion by four bits - '9', 'A' - 'F' consisting of numbers and letters of the string. To send a message to form UCS2 code for transmission.

\subsubsection{Chinese Unicode code}

We got through the UCS2 encoding Chinese Unicode code, and then they can be prepared to send a PDU string. On the surface, PDU string is ASCIl string, also by the 'O' - '9', 'A' - ' $F$ ' these Numbers and letters. They are 8-byte hexadecimal, decimal or BCD code. PDU string in addition to containing the send message itself, also contains a lot of information, other parameters such as the service center number and target number and encoding, etc.

\section{Some examples:} 0051000BA13108086406F600F5A7850B05040B8423F_ 0000303010129060603 AE81EA8DCA02056A0045C6080C0332313 $12 e_{-}$

3133362e3135332e33302f776170707573682f70757368496e64657 $82 \mathrm{e}$

6a73̄703f7075736849643d303530353131313431363035323100010 3E8A

FB7E782B9E587BBE4BBA5E4B88BE993BEE68EA5E88EB7E58F9 6E5BDA9E4BFA1E58685

E5AEB9000101_ 
As WAP PUSH string can be successfully sent, including a description of the characters and WAP page address. Specifically as follows

Length 00 SMSC address information 00 set SMS center number, PDU string on the phone with "SMSC Address Format" section and the "SMSC Address" section will be omitted.

51 basic parameters (TP-MTI / VFP) is not required to send a reply.

00 Message reference value (TP-MR)

$\mathrm{OB}$ length of the other phone

A1 represents the destination format for domestic format

$3108086406 \mathrm{~F} 6$ destination address, fill ' $\mathrm{F}$ ' to make up after even bit parity exchange

00 protocol identification (TP-PID) is an ordinary GSM type, point the way

F5 user information encoding (TP-DCS)

A7 validity (TP-VP)

85 User Information Length (TP-UDL)

The total length of the head OB WAP PUSH

05040B8423F0 means that the following is a WAP PUSH

00 is Concatenated Short Messages

03 Length

03 reference number

01 that is divided into an SMS

The current number of 01 packets

29060603AE81EA8DCA WSP

02 flag

05 - // WAPFORUM // DTD SI 1.0 // EN

6 A UTF-8

00 mark beginning

$45<$ si>

C6 <indication>

$08<$ action=signal-high>

OC href="http://

03 start of the string

3231312e3133362e3135332e33302f776170707573682f

$70757368496 \mathrm{e} 6465782 \mathrm{e} 6 \mathrm{a} 73703 \mathrm{f} 7075736849643 \mathrm{~d} 3035303531313$

134313630353231 URL
00 URL string End

$01>$

03 Start of Description string

E8AFB7E782B9E587BBE4BBA5E4B88BE993BEE68EA 5E88EB7E58F96E5BDA9E4BFA1E58685E5AEB9 description string 00 Description string end

$01</$ si $>$

$01<$ /indication>

As can be seen from the above analysis, WAP PUSH can be used as a special message to send the contents of WAP PUSH packet is actually sent through the PPG principle with XML gateway is the same. But after a compressed format called WBXML after compression, this format will be marked with the code number to represent. However WBXML abbreviated mark is divided into two parts, one is for all types of XML are common, the other part is a different type of XML has a different interpretation. Under When a user receives such information, the client phone supports WAP case, you can directly access to the information loaded WAP site address so that the server has reached a userfriendly business promotion purposes. Since in UCS2 encoding, the maximum number of characters you can send short messages is 140 bytes, the total number of characters in the WAP PUSH push URL and description text is 140 , so the description text limits and push URL length.

WAP PUSH technology combines the characteristics and technical advantages of PUSH and mobile communication services, has a good prospect. This technology has a transmission distance without having to build a wide network of independent coverage and other characteristics, can be widely used in smart meter reading gas meters, data management and monitoring.

\section{REFERENCES}

1. Chen Hailin, Dong Tao. Development and Application of Diaphragm gas meter (SMS) and WAP. Electronic Industry Press, 2002.12.

2. Cui Yan. Short Message Service System. Xi'an University of Electronic Science and Technology, 2013. 